\title{
Optimal Selection of the Reference Potential Probe Point in DCPD Real-Time Monitoring of Crack Growth Rate
}

\author{
He Xue $\mathbb{D}^{1},{ }^{1}$ Siyu Gou $\left(\mathbb{D},{ }^{1}\right.$ Chenqiang Ni, ${ }^{1}$ Liang Zhang, ${ }^{1}$ Shuai Wang $\mathbb{D}^{1,2}$ and Youjun Zhao ${ }^{1}$ \\ ${ }^{1}$ School of Mechanical Engineering, Xi'an University of Science and Technology, Xi'an 710054, China \\ ${ }^{2}$ Department of Mechanical and Aerospace Engineering, Brunel University, London, Uxbridge UB8 3PH, UK \\ Correspondence should be addressed to Siyu Gou; 18205019028@stu.xust.edu.cn
}

Received 30 August 2020; Revised 6 November 2020; Accepted 9 November 2020; Published 23 November 2020

Academic Editor: Paolo Andrea Carraro

Copyright (C) 2020 He Xue et al. This is an open access article distributed under the Creative Commons Attribution License, which permits unrestricted use, distribution, and reproduction in any medium, provided the original work is properly cited.

Quantitatively monitor the crack growth rate of material stress corrosion cracking (SCC) in an autoclave that simulates a hightemperature and high-pressure water environment, and the direct current potential drop (DCPD) method is the main method. Since the DCPD method tests micro-nano-voltage drop signals, the monitoring signal is weak and easy to be interfered by the environment. To reduce and balance the error caused by the temperature drift and other factors to the monitoring accuracy, it is very important to reasonably select the position of the reference potential probe point. In this study, genetic algorithm (GA), finite element method (FEM), and experimental analysis are used to optimize the position of the reference potential probe point of the compact tensile (CT) sample. Finite element method is used to analyze the electric potential field of the compact tensile sample, a mathematical model of the measurability and crack independence of the reference potential difference are constructed, genetic algorithm is used to find the optimal reference potential difference (RPD) probe point position, and finally, the crack monitoring experiments are performed to evaluate the feasibility of algorithm optimization results. The results indicate that the RPD measured at the current input point and the upper right position of the CT sample can provide the maximum compensation for the potential on both sides of the crack and make the performance of the monitoring signal optimal.

\section{Introduction}

In an autoclave that simulates the high-temperature water environment of nuclear power, monitoring of the crack growth rates is one of the important tasks in the selection of the structural materials and safe evaluation of nuclear power [1]. Because of the special environmental constraints of the autoclave, there are few methods to monitor the crack growth in real time. The potential drop method is currently one of the most widely used and most important methods, which reflects the change of the crack length by measuring the potential drop at both ends of the CT sample with a constant current [2]. Potential drop method is divided into the direct current potential drop (DCPD) method and alternating current potential drop (ACPD) method. ACPD is susceptible to noise caused by external factors such as unstable current and improper installation of equipment [3], while DCPD is more suitable for monitoring crack initiation and propagation of samples in a laboratory environment [4].
The experimental period of the stress corrosion cracking in the high-temperature water environment is normally long [5], and the entire process of crack monitoring needs to be carried out with nanometer test accuracy. Since the potential difference signal that characterizes the crack length is extremely small, temperature fluctuations will cause the resistivity of the sample to change, which will make great interference with the potential difference signal [6]. Recently, neural network [7], machine learning [8], and reference potential method [9] are used to suppress the temperature drift; neural network and machine learning usually adoptedbig database to build parameter models, and a look-up table can be obtainedafter training; eventually, it realized the correction and compensation of temperature drift, but these two methods can not solve the fluctuation of current and voltage problems. However, the reference potential difference method has a good effect on suppressing the phenomenon of current leak and changes in electrical 
conductivity. It selects the potential difference at any two probe points on the sample as the reference potential difference (RPD) which corrects and compensates the main potential difference reflecting the growth of the crack to eliminate the fluctuation of the potential difference caused by the temperature drift. Additionally, the position of the RPD probe points is diversified, and the compensation amount of the reference potential difference to the main potential difference will be different; therefore, it is vital to choose the position of the RDP probe point reasonably to eliminate the influence of other factors on the crack measurement. Ni and Xue [10] used the FEM to study the potential field distribution of CT samples and analyzed the selection of the probe point position from sensitivity, signal amplitude, and measurement error. Based on the FEM, Hu [11] recently proposed that the ratio between the reference potential and the main potential is dimensionless and then obtained the relationship between crack length and voltage drop of the three-point bending sample. Campagnolo et al. $[12,13]$ adopted a three-probe potential drop method to assess the crack configuration and location, and the calibration curves reporting the potential drop change as a function of the crack depth are derived utilizing 3-dimensional electrical finite element analyses; finally, it has been validated by systematic comparison with experimental results. Spitas et al. [14] recently proposed a method that uses the finite element analysis to simulate the change of the potential within stretching and compression of the sample through real-time assessment of whether the length and direction of the crack have changed, and it was found that the center electrode was sensitive to crack length and insensitive to crack angle.

In this study, a novel method is proposed based on the genetic algorithm to obtain the optimal reference potential difference and the probe point position that meets the best measurability, and crack independence is sought. Finally, a crack monitoring experiment is operated in an autoclave environment, and the accuracy, resolution, and stability of the monitoring data are analyzed at the optimal probe point position.

\section{Model Establishment}

2.1. Theoretical Model of the RPD. The theoretical model is established based on the measurability of the potential difference and the crack independence. For a series of operations such as selection, variation, and crossover of potential populations, eventually, the optimal position of the probe point is sought. The theoretical model is established as follows:

$$
\begin{aligned}
U & =\left\{U_{n}\left|U_{n}=\right| X_{i j}-X_{p q} \mid, \quad U_{n}>U_{t}\right\}, \\
\Delta U & =\left\{\Delta U_{m n}\left|\Delta U_{m n}=\right| U_{m}-U_{n} \mid, \quad \Delta U_{m n}<U_{s}\right\},
\end{aligned}
$$

where $U$ is the RPD between two probe points and $\Delta U$ is the difference between the RPD at the same two probe points for different crack lengths. To meet the resolution of crack monitoring instruments, the constraints are defined as the following formulas:

$$
\begin{aligned}
& U_{t}=0.5 U_{o}, \\
& U_{S}=0.1 U_{n},
\end{aligned}
$$

where $U_{t}$ is the threshold of the RPD and $U_{s}$ is the threshold of the difference between the RPD, and the unit is $\mu \mathrm{V}$.

Except for the loading hole, probe points of the reference potential are distributed along the path, as shown in Figure 1. Lower left of the path is defined as the initial point of the row and column, $i$ or $p$ is the row coordinate, and $j$ or $q$ is the column coordinate; however, after the selection of $i$ and $j$ coordinates, $p$ and $q$ coordinates can be selected among the remaining coordinate points, which are shown in formulas (5) and (6). Besides, the probe points are defined as $K, K 1$, and $K 2$ which refer to different probe points, and the position of any two different probe points is defined as $K_{i j}$ or $K_{p q}$. Meanwhile, $n$ refers to the number of $\mathrm{RPD}$, and $m$ and $z$ refer to different cracks. With the crack growing, the value of the RPD is also different, and the parameter $n$ changes with the crack length $a_{n}$.

The measurability refers to the value of the RPD between two probe points. The larger the reference potential difference, the better the measurability, and the smaller the reference potential difference, the worse the measurability. Therefore, the measurability model is defined as

$$
U_{n}=\left|X_{i j}-X_{p q}\right|, \quad(i \neq p, j \neq q) .
$$

Restrictions:

$$
\begin{aligned}
& U_{n}>0.01 U_{o}, \\
& 0 \leq i<p \leq 31, \\
& 0 \leq j<q \leq 15,
\end{aligned}
$$

where $U_{n}$ is the RPD between two probe points on the $n$-th crack length, in other words, for the same crack length, the potential difference between one probe point with coordinate $(i, j)$ and another probe point with coordinate $(p, q) . U_{o}$ is the main potential difference (MPD) and $X_{i j}$ and $X_{p q}$ are the potentials of any two probe points on the samples with $n$ th crack length.

Crack independence refers to the difference of the RPD between the same two probe points with different crack lengths, which reflects the change of the RPD at the same two probe points. Contrary to the measurability, the smaller the difference, the better the crack independence, and the larger the difference, the worse the crack independence. Consequently, the crack independence model is described as follows:

$$
\Delta U_{m n}=\left|U_{m}-U_{n}\right|=|| Y_{i j}-Y_{p q}|-| X_{i j}-X_{p q} \|,
$$

where $\Delta U_{m}$ is the RPD between two probe points on the $m$ th crack length, $\Delta U_{n}$ is the RPD between two probe points on the $n$-th crack length, $\Delta U_{m n}$ is the difference between the RPD of the $m$-th crack length and the $n$-th crack length, and $Y_{i j}$ and $Y_{p q}$ are the potentials of any two points on the samples with $m$-th crack length. 


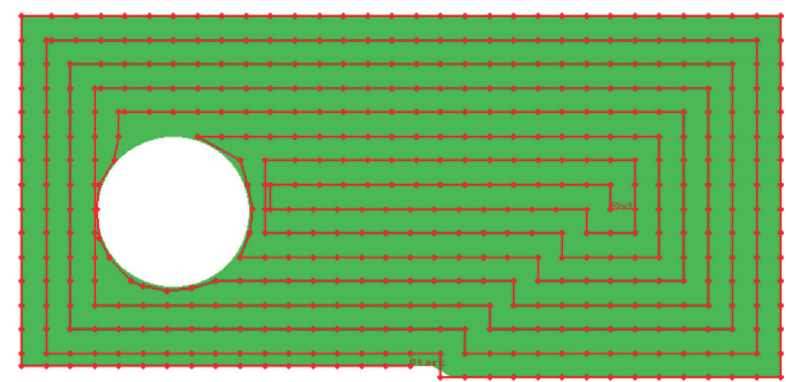

FIgURE 1: The extraction path of reference potential probe points.

The distance at any two probe points is defined as

$$
d_{n}=\sqrt{(i-p)^{2}+(j-q)^{2}}
$$

where $d_{n}$ is the distance between any two probe points.

2.2. Finite Element Model. Finite element software Abaqus is an effective numerical analysis method; it is widely used to solve continuity problems such as heat conduction, electromagnetic fields, and fluid mechanics. Therefore, it is selected to analyze the potential field of $0.5 \mathrm{~T}$-CT samples in this paper. According to the path of the CT sample, the position of reference potential probe points is selected. Generally, geometry and the current input point of the CT sample are symmetrical to the crack, with a constant electrical current of $1 \mathrm{~A}$ introduced from point $\mathrm{A}$ at the upper left of the sample. Sample ligament which is from the crack tip to the right side is set to the position of zero potential, see the red area in Figure 2. The length and width of the CT sample are $1.25 \mathrm{~W}$ and $1.2 \mathrm{~W}$, the diameter of the loading hole is $0.25 \mathrm{~W}$, and the length of the initial crack is $a_{0}$. The geometric parameters and properties of the materials are shown in Table 1.

The crack length refers to the distance from the loading hole to the crack tip according to the ASTM 399 standard, and the length of the initial crack is set to $a_{0}=12 \mathrm{~mm}$. Using an eight-node quadratic thermoelectric coupling quadrilateral element (DC2D8E), the total number of grids is 8786 , the mesh is created automatically from software, and it is refined by 0.01 times of the global grid for the electrical current input area and crack tip area, as shown in Figure 3.

As the length of the crack increases, the position of the zero potential gradually decreases, and simultaneously, the distribution of the potential field of the CT specimen is also changing. The total length of crack growth is $4 \mathrm{~mm}$, the reference potential of the CT specimen is extracted for each $0.5 \mathrm{~mm}$, a total of 8 extractions are performed, and the potential field distribution of CT specimens is shown in Figure 4 . The result shows that the potential value at the electrical current input point is the largest, and the potential value near the crack tip is the smallest. Eventually, equipotential surfaces will be formed along both sides of the crack.

According to the finite element analysis, cracks on the CT specimen have the same lateral expanded state along the thickness direction. Therefore, the CT specimen with the 2D

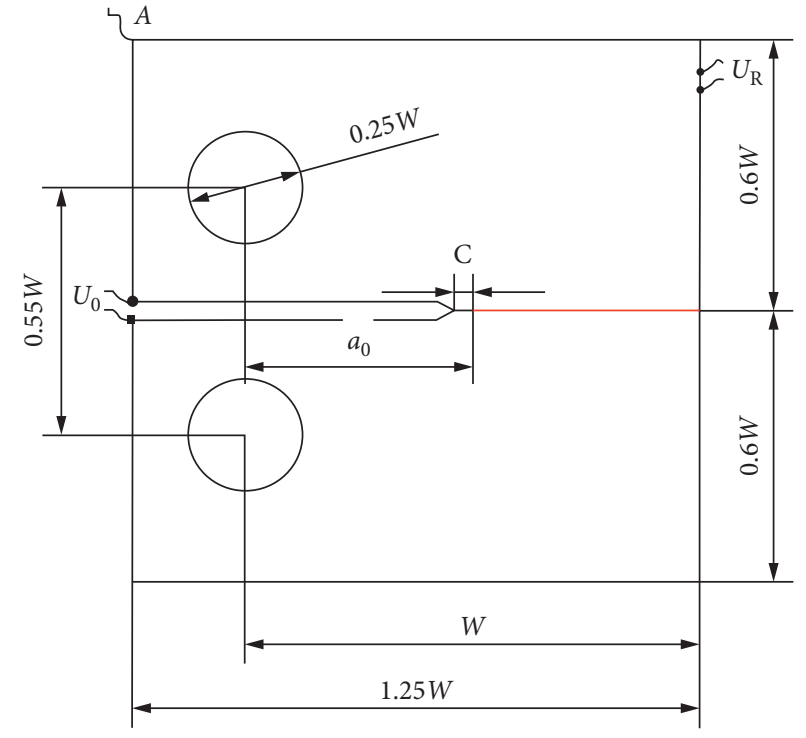

FIGURE 2: CT specimens' geometry and shape.

plane is selected as the research object to extract all the reference potentials. The crack tip near the ligament is selected as the initial reference potential probe point. A total of 494 probe points are placed in sequence along the counterclockwise path of the specimen at a $1 \mathrm{~mm}$ grid node, as shown in Figure 5. Since the voltage value at current input point A is gigantic, the lower left of the CT specimen is the position of the main potential probe point. Meanwhile, to ensure the positional accuracy of the probe points and load easily, the unreasonable reference potential probe points of the current input point, the main potential, and the loading hole should be removed.

\section{Genetic Algorithm Optimization for RPD Probe Points}

3.1. Multiobjective Function of GA. Genetic algorithm is a method to search the optimal solution by imitating natural evolution in the biological world. Compared with other optimized algorithms, GA has the characteristics of fast convergence and global optimization. It is widely used to deal with complex optimization problems [15], and the basic operation of GA is to establish a suitable target function. After coding, decoding, and other operations, the population is also evaluated. Ultimately, multiple iterations are performed to find the optimal solution through genetic operations such as selection, crossover, and mutation [16-18], and the realization process of the genetic algorithm is shown in Figure 6.

GA optimizes the position of the probe points, which is realized by optimizing the RPD to obtain the optimal RPD value. Since the numbers of RPD obtained by the exhaustive search are numerous, there are no rules to follow. Therefore, GA is selected to optimize the objective function to obtain optimal RPD and RPD position.

The objective function must meet the two conditions of maximum measurability and minimum crack 
TABLE 1: Material properties and geometric parameters of the specimens.

\begin{tabular}{lccccccc}
\hline Material & $\sigma_{0.2}(\mathrm{MPa})$ & $E_{0}(\mathrm{GPa})$ & $v$ & $\sigma(\mathrm{S} / \mathrm{m})$ & Crack thickness B $(\mathrm{mm})$ & Initial crack $a_{0}(\mathrm{~mm})$ & $W(\mathrm{~mm})$ \\
\hline $316 \mathrm{~L}$ & 221 & 193 & 0.3 & 1.45 & 12.5 & 12 & 25 \\
\hline
\end{tabular}

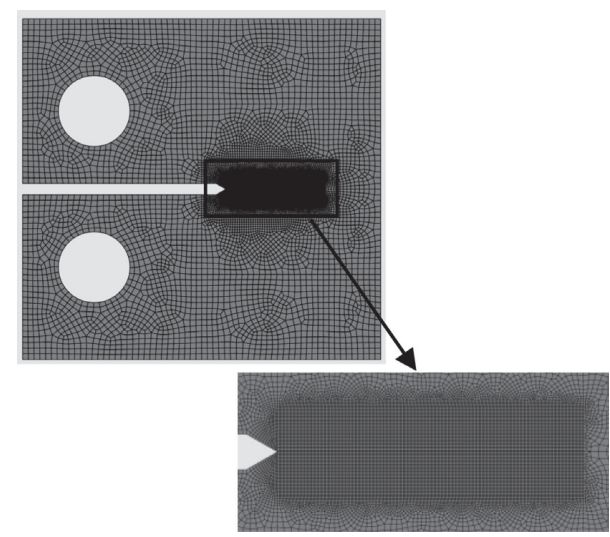

FIgURE 3: Global and local mesh of CT specimens.

independence. To make the RPD satisfy the maximum measurability, making the RPD in formula (3) equal to a constant $C$, its formula is

$$
\max U_{n}=\lim _{n \longrightarrow \infty}\left|X_{i j}-X_{p q}\right|=C .
$$

Formula (10) can be obtained by squaring and shifting the terms on both sides of formula (9), and the fitness function is deduced to obtain formula (11):

$$
\begin{gathered}
X_{i j}^{2}-2 X_{i j} X_{p q}+X_{p q}^{2}-2 C X_{i j}+2 C X_{p q}+C^{2}=0 \\
F\left(X_{i j}, X_{p q}, C\right)=X_{i j}^{2}-2 X_{i j} X_{p q}+X_{p q}^{2}-2 C X_{i j}+2 C X_{p q}+C^{2} .
\end{gathered}
$$

To maximize the objective function, the following conditions should be satisfied:

$$
\begin{gathered}
\frac{\partial F}{\partial X_{i j}}=0, \\
\frac{\partial F}{\partial Y_{i j}}=0, \\
\frac{\partial F}{\partial C}=0 .
\end{gathered}
$$

Since the reference potential difference must satisfy the minimum crack independence, therefore, when the value of $m$ and $n$ tends to infinity, the difference between the RPD of different crack lengths at the same probe point gradually equals to zero; formula (13) can be obtained by transforming formula (7):

$$
\min \Delta U_{m n}=\lim _{\substack{m \longrightarrow \infty \\ n \longrightarrow \infty}}\left(\left|X_{m}-X_{n}\right|-\left|Y_{m}-Y_{n}\right|\right)=0 .
$$

Both sides of formula (13) are squared, and the absolute values are removed as follows:

$$
X_{m}^{2}-2 X_{m} X_{z}+X_{z}^{2}-2 X_{m} Y_{m}+2 X_{m} Y_{n}+2 X_{n} Y_{m}-2 X_{n} Y_{n}+Y_{m}^{2}-2 Y_{m} Y_{n}+Y_{n}^{2}=0
$$

Let $C_{i}=X_{m}^{2}+X_{n}^{2}+Y_{m}^{2}+Y_{n}^{2}$ and

$$
-2 X_{m} X_{n}-2 X_{m} Y_{m}+2 X_{m} Y_{n}+2 X_{n} Y_{m}-2 X_{n} Y_{n}-2 Y_{m} Y_{n}+C_{i}=0
$$

The fitness function is defined as follows:

$$
M\left(X_{m}, X_{n}, Y_{m}, Y_{n}\right)=-2 X_{m} X_{n}-2 X_{m} Y_{m}+2 X_{m} Y_{n}+2 X_{n} Y_{m}-2 X_{n} Y_{n}-2 Y_{m} Y_{n}+C_{i}
$$

To minimize formula (16), the fitness function should satisfy the conditions as follows: 


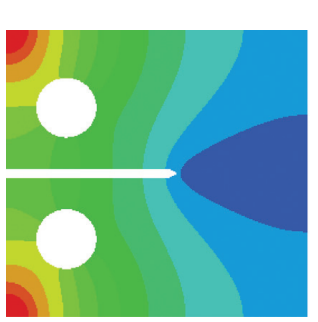

(a)

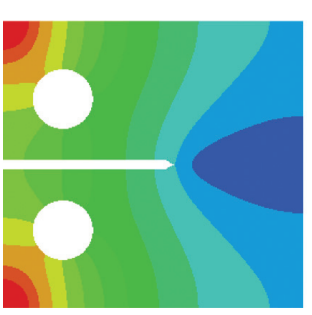

(d)

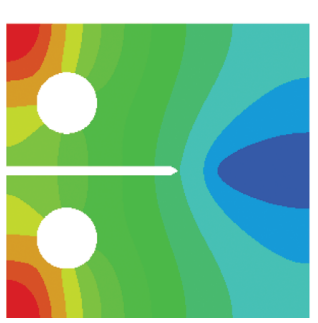

(g)

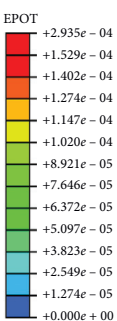

$+1.274 e-05$

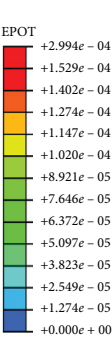

$+1.2744-0.00$
$+0.000 e+0$

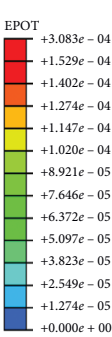

$+1.274 e-05$
$+0.000 e+00$

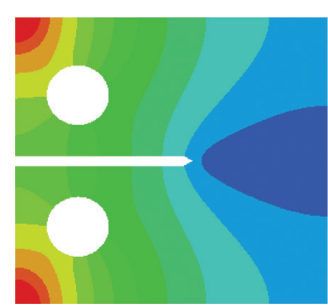

(b)

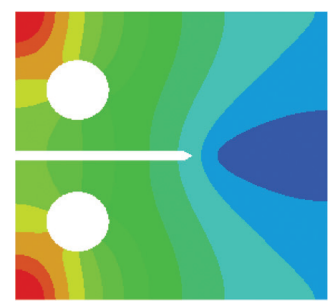

(e)

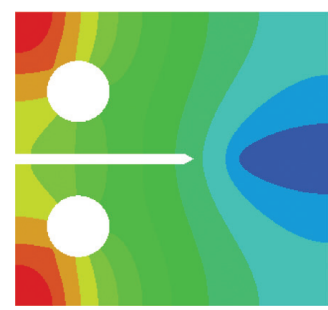

(h)
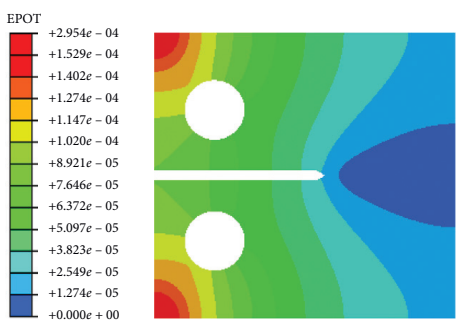

(c)
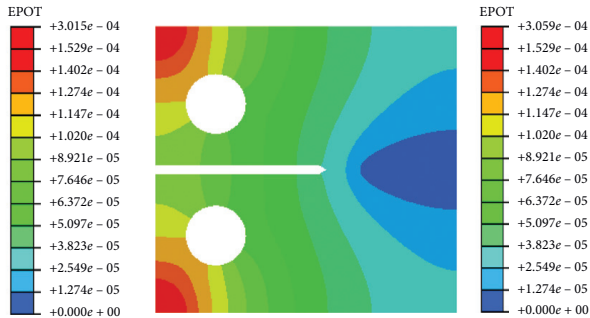

(f)
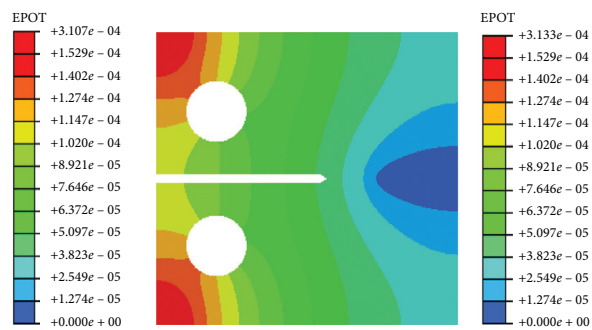

(i)

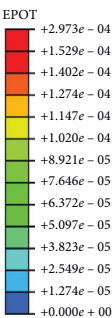

$-1.2740-0.5$
Figure 4: The potential field distribution of CT specimens: (a) $a_{0}=12 \mathrm{~mm}$, (b) $a_{1}=12.5 \mathrm{~mm}$, (c) $a_{2}=13 \mathrm{~mm}$, (d) $a_{3}=13.5 \mathrm{~mm}$, (e) $a_{4}=14 \mathrm{~mm}$, (f) $a_{5}=14.5 \mathrm{~mm}$, (g) $a_{6}=15 \mathrm{~mm}$, (h) $a_{7}=15.5 \mathrm{~mm}$, and (i) $a_{8}=16 \mathrm{~mm}$.

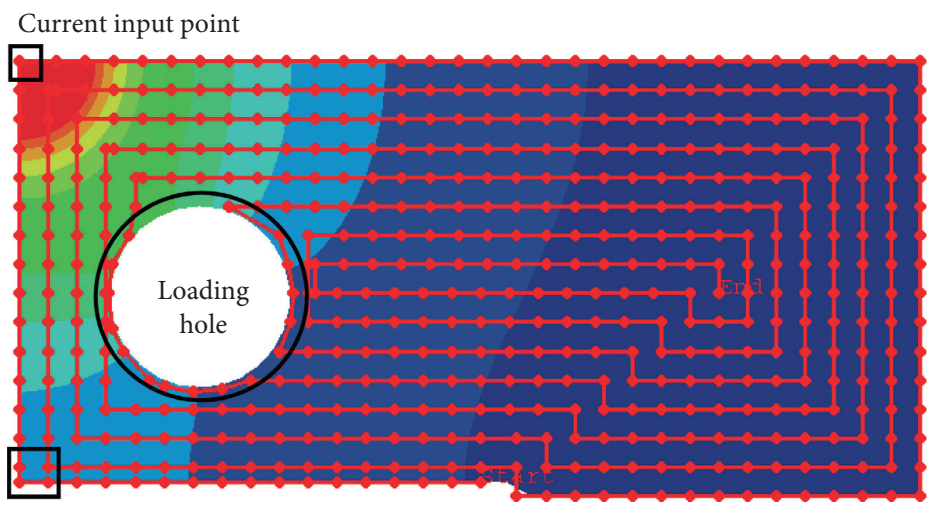

EPOT

Main potential

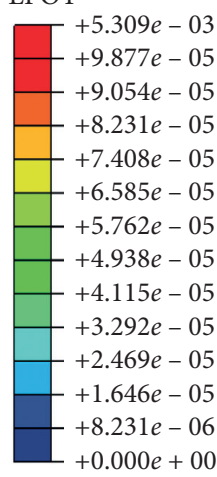

$+0.000 e+00$

Figure 5: The distribution of reference potential probe points of the CT specimen. 


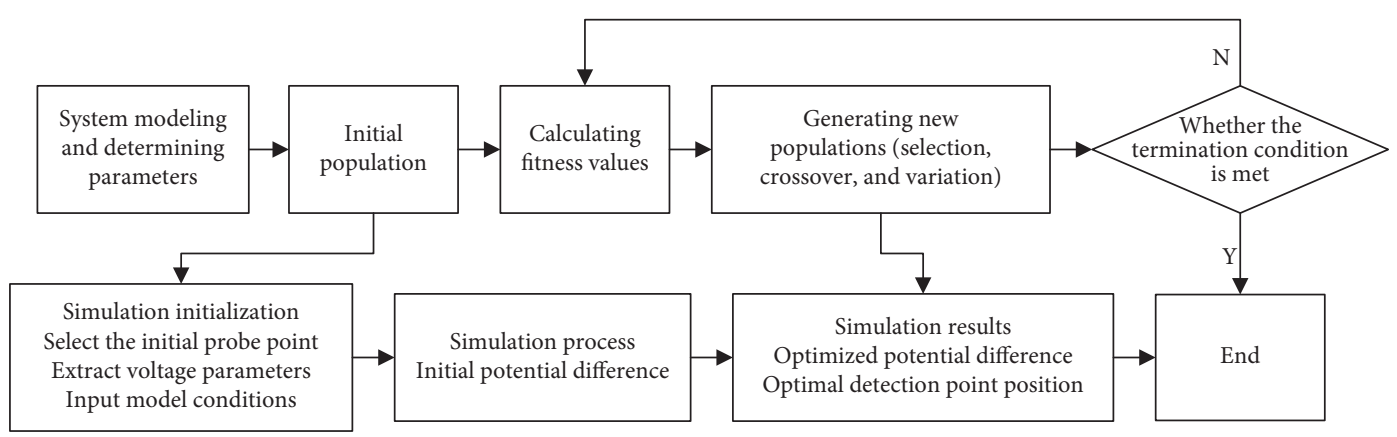

FIGURE 6: Flowchart of GA.

$$
\begin{gathered}
\frac{\partial M}{\partial X_{m}}=0, \\
\frac{\partial M}{\partial X_{n}}=0, \\
\frac{\partial M}{\partial Y_{m}}=0, \\
\frac{\partial M}{\partial Y_{n}}=0 .
\end{gathered}
$$

3.2. Optimization Process of GA. The length of the first crack propagation $a_{1}=12.5 \mathrm{~mm}$ is selected as the research object, and the implementation process of GA is described briefly as follows.

3.2.1. Coding and Decoding. According to the position coordinates of the probe point on the CT specimen, a binary coding method is adopted to encode and decode the RPD. By selecting nine RPDs as a representative and a chromosome supposed as $[2 \cdot 3 \cdot 2 \cdot 1 \cdot 1 \cdot 2 \cdot 2 \cdot 3 \cdot 1]$, the specific implementation process is shown in Figure 7 , and the coding is based on the size of the population and the value of the objective function. The accuracy of the solution is defined through constraints, and then the actual value is converted into a binary code. Similarly, the decoding process is equivalent to the inverse operation of encoding, which converts the gene string value into the actual value in the solution space. If the objective function has a range of value $\left[a_{i}, b_{i}\right]$ and divides the solution space of $x$ into 99999 aliquots, it satisfies

$$
\left(b_{i}-a_{i}\right) \times(1 E+4) \leq 2^{n i}-1 .
$$

The solution process is as follows:

$$
X_{i}=a_{i}+\operatorname{decimal}(0001010 \ldots 01) \times \frac{b^{i}-a^{i}}{2^{n i}-1} .
$$

3.2.2. Initial Population Generation. The generation of the initial population primarily involves in determining
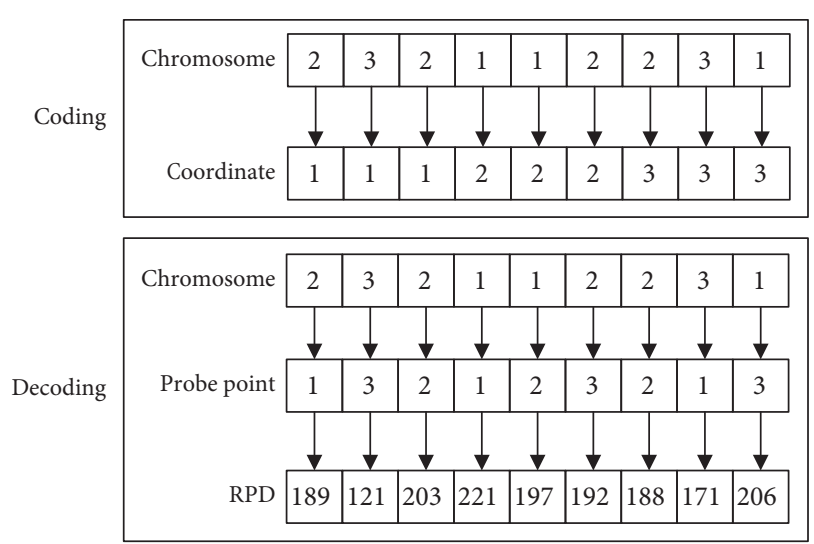

FIGURE 7: The specific implementation process of encoding and decoding.

population size and calibrating initial population parameters. When the selected initial population parameter range differs greatly from the true value, it often has a slower convergence rate, and even nonconvergence. Therefore, a reasonable selection of the parameter range of the initial population is needed, which affects computational accuracy and efficiency [19]. It is based on the size of the CT specimen with calibrated parameters and the number of initial RPD obtained by the exhaustive search to define the value range of uncalibrated parameters. When the crack length is $a_{1}$, the number $n$ of RPD obtained by the exhaustive search is 65621 . To find the global solution faster, the size of the initial population is selected as 100 .

3.2.3. Selection. The reference potential difference fitness function can be used for new individual selection. The probability of the RPD which has been selected is calculated by summing and ranking the probability one by one; if it satisfies less than the fitness function value, the probability will be selected, until the condition is not confirmed. Afterward, the range of the fitness function is expanded, making multiple selections, and finally obtaining an optimal RPD individual.

3.2.4. Crossover and Mutation. After the selection operation, the optimal and eliminated RPD individuals are removed; meanwhile, the remaining RPD individuals are 
sorted according to the fitness value. Ultimately, the crossover operation is performed by using roulette to obtain a new population, and new individuals are generated from those who have been selected for superiority to expand the number of populations [20].

The new population of the reference potential difference has been obtained by performing crossover and mutation operations, and the range of the mutated rate [21] is 0.0001 $\sim 0.1$. Generally, for the population with the larger objective function, a smaller mutated rate is selected, and for populations with smaller objective functions, a larger mutated rate is selected [22].

The crossover and mutation formulas are as follows:

$$
\begin{aligned}
p_{c} & =0.7+(0.9-0.7) \operatorname{rand}(m, n), \\
p_{m} & =0.01+(0.05-0.01) \operatorname{rand}(m, n) .
\end{aligned}
$$

In the formula, $p_{c}$ is the probability of crossover, $p_{m}$ is the probability of mutation, and rand $(m, n)$ is a random number between 0 and 1 that generates $m$ rows and $n$ columns.

Eventually, the process of the GA operation is repeated until it stops when the optimal solution of the objective function is obtained. In the implementation process of GA, the range of the objective function is $[0,65621]$, the population size $N$ is 100 , the chromosome length $X_{i}$ is 10 , the number of iterations is 1000 , the crossover probability $p_{c}$ is 0.8 , and the mutation probability $p_{m}$ is 0.1 .

\section{Experimental Environment}

The purpose of this experiment was to verify the effectiveness and accuracy of GA, optimizing the position of RPD probe points. Figure 8 shows the experimental platform for DCPD to monitor crack growth. To facilitate the comparison of test results, the alphabets $\mathrm{Q}, \mathrm{R}, \mathrm{M}$, and $\mathrm{N}$ are used, respectively, to denote the combination of the two positions of RPD probe points, which are near the current input and the upper right side of the specimen (combination Q), near the current input and crack tip (combination R), near the current input and the bottom right side (combination $M$ ), and the upper right side and the lower right area (combination $\mathrm{N}$ ); the overall experimental positions of probe points are shown in Figure 9. Eventually, the crack monitoring experiment is performed, and the test data of the apparatus are comprehensively analyzed regarding the stability, measurability, and crack independence to judge whether they are consistent with the optimized results of GA.

4.1. Experimental Devices. The experiment is composed of three parts; an autoclave vessel is used to provide a hightemperature environment for crack growth. Stress-corrosion fatigue tensile machine loads the CT specimen to achieve crack propagation. Meanwhile, the DCPD crack monitoring system is used to obtain the potential value of probe points in real time. The DCPD crack monitoring system includes a high-performance power supply, a nanovoltmeter, a multiplex switch, and a computer.

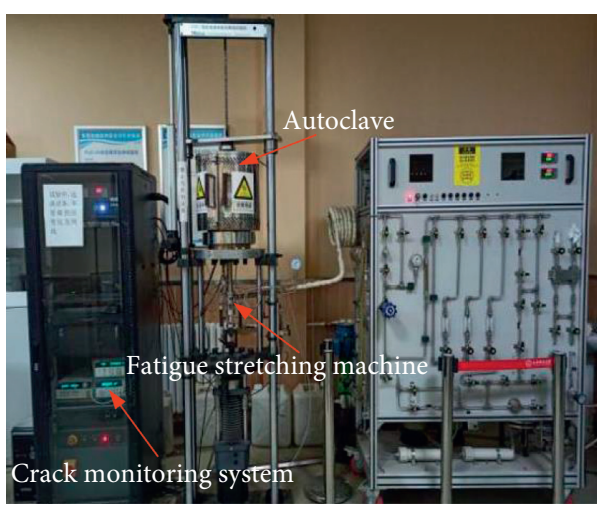

Figure 8: Experimental device for crack monitoring.

The high-performance power supply is used to provide a constant current to the CT specimen, the nanovoltmeter is used to measure the potential drop, and the multiplex switch is used to switch between the main potential signal and the reference potential signal. These devices are finally connected to the computer through the GPIB bus and work under the control of test software; the main equipment and models used in the experiment are shown in Table 2.

4.2. Experimental Parameter Settings. The experiment is carried out in an autoclave with a high temperature of $300^{\circ} \mathrm{C}$, the current input of high-performance power supply is set to $0.15 \mathrm{~A}$, and the voltage input is set to $0.1 \mathrm{~V}$. To take into account the measurement speed and measurement accuracy, the integration time of the nanovoltmeter is set to 10 power line cycles, the resolution of the measurement result is $1 \mathrm{nV}$, and the monitoring process takes 2.6 seconds. Besides, the type of fatigue load is sine wave, the peak force is $8000 \mathrm{~N}$, the valley force is $3000 \mathrm{~N}$, the loading frequency is $1 \mathrm{~Hz}$, and the experiment lasts for about 10 hours. Afterward, a constant amplitude load was used to keep the load at $8000 \mathrm{~N}$ to make the crack grow slowly, and the experiment lasted about 300 hours in total.

4.3. Experiment Process. With the selected CT specimen as an experimental object, it is usually placed in an autoclave, and the initial crack length prefabricated by wire cutting is $12.5 \mathrm{~mm}$. Since the CT specimen is symmetrical, the probe points of the current input and output are located on both sides of the specimen, and the probe points of the main potential are located on both sides of the crack. On different temperatures, the speed of the crack growth is different. Due to the slow rate of crack growth, which is approximately $9.26 E-7 \mathrm{~mm} / \mathrm{s}$, the experimental data with a large temperature difference during the whole day are selected for comparison. When the instrument starts collecting data, it needs to be warmed up for an hour; otherwise, the initial data will fluctuate greatly. To accurately reflect the crack independence, a large potential difference between the initial and final crack length is selected for comparison. The measurability and crack independence at different probe points are analyzed and compared, which verify the results of GA feasibility of the optimization. Reference potential can 


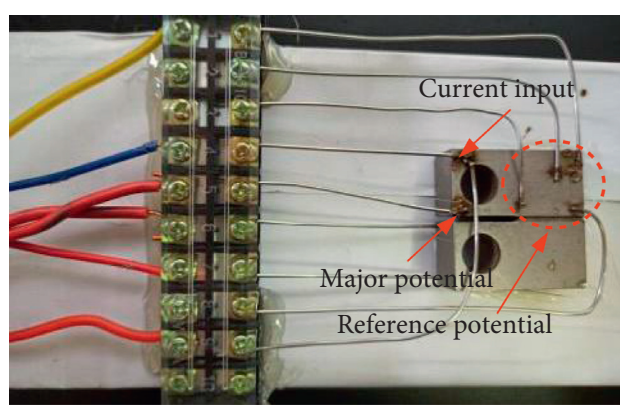

FIgURE 9: The position of probe points.

provide compensation for the main potential on both sides of the crack; therefore, it can be judged whether the reference potential probe points are optimal according to the stability, accuracy, and resolution of the main potential monitoring signal.

\section{Results and Discussion}

5.1. FEM to Obtain Reference Potential. The crack length $a_{n}$ is $12,12.5,13,13.5, \ldots, 16$. With the increase of crack length, the potential value at the same probe points varies. The FEM is used to extract the potential value of the probe points since the voltage signal characterizing the crack is very weak. When the crack grows $0.5 \mathrm{~mm}$ each time, there is an analogous potential of the CT specimen at the same probe points. Removing the unreasonable potential value at the current input point for different crack lengths, the distribution of the reference potential regarding the CT specimen is shown in Figure 10. M refers to the coordinate of the probe point with the largest potential value.

As shown in Figure 10, the reference potential values of probe points are periodically decreasing fluctuation, which appears to have a trend of increasing first and then decreasing in each cycle, and potentials are symmetrically distributed at both sides of the maximum. According to the number $K$ of the probe point, the closer the probe point is to the current input, the larger the potential value is. With the increase of the length of the crack, the potential value of the probe point gradually becomes larger.

\subsection{Optimization Results of $G A$}

5.2.1. The Measurability of the RPD. For the study of RPD measurability, the simulation experiment is based on the crack length $a_{1}=12.5 \mathrm{~mm}$. FEM extracts the potential value of probe point $K$, and the number $n$ of the RPD of any two probe points is 65621 by the exhaustive search method, and the distribution of the RPD at different probe points of CT specimens is shown in Figure 11(a). The two horizontal coordinates show the number of the probe points $K 1$ and $K 2$, and the vertical coordinate shows the value of RPD $U_{n}$. Since the distribution of the RPD is relatively scattered, it is difficult to obtain directly the position of the optimal probe points of the CT specimen. Therefore, the RPD measurability model regarding GA is constructed. After 1000 iterations, the value of the RPD after optimization is rearranged, and the optimization results of the RPD for different crack lengths are shown in Figure 11(b).

It can be seen in the figure that the distribution of the optimized RPD value has a monotonous decreasing trend; meanwhile, RPD has the same trend for different crack lengths, which instructs the growth of the crack length which has little effect on the measurability of the RPD. It is well known that the closer the crack, the worse the crack independence, and with the increase of the length of the crack, the number $n$, the value of $U_{n}$, and the coordinates $K 1(i, j)$ and $K 2(p, q)$ of the two probe points of the RPD optimized by the GA are shown in Table 3 .

From the position and coordinates of the optimal probe points in Table 2, as the length of the crack grows, the value of the optimal RPD after GA optimization gradually increases. The two probe points where the RPD meets the greatest measurability are concentrated near the current input and crack tip (combination $\mathrm{R}$ ) and near the current input and the upper right side of the specimen (combination Q).

\subsubsection{The Crack Independence of the RPD Difference.} The crack independence model is based on the difference value between the RPD at the same probe points with different crack lengths. Since there are too many combinations of two different crack lengths, to reflect the crack independence intuitively, the research object of crack-independence is divided into 5 groups, which are the crack length $13 \mathrm{~mm}$ and the crack length $13.5 \mathrm{~mm}$, crack length $13 \mathrm{mmand}$ the crack length $14 \mathrm{~mm}$, crack length $13 \mathrm{~mm}$ and the crack length $14.5 \mathrm{~mm}$, cracklength $13 \mathrm{~mm}$ and the crack length $15 \mathrm{~mm}$, crack length $13 \mathrm{~mm}$ and the crack length $15.5 \mathrm{~mm}$, crack length $13 \mathrm{~mm}$ and the crack length $16 \mathrm{~mm}$. A mathematical model of crack independence is established to obtain the difference value of the RPD, and the distribution is shown in Figure 12(a). It is based on the measurability of the RPD. A genetic algorithm model of crack independence is constructed. The distribution value of the difference between the RPD after optimization is rearranged, and the optimal result is shown in Figure 12(b).

It can be seen from the figure that two different crack lengths and the optimal difference between the RPD at the same probe points are different; however, the numerical distribution trend of the difference between the RPD is almost the same. With the increase of the potential difference between the two crack lengths, the maximum value of the difference between the RPD at the same probe points gets larger gradually. According to the serial number $n$ corresponding to the difference value $\Delta U$ of the optimal RPD, the optimization of the genetic algorithm finds the position of two probe points $K 1(i, j)$ and $K 2(p, q)$ that satisfy the optimal crack independence as shown in Table 4.

Comprehensive analysis of the GA gives optimized results of RPD measurability and crack independence for all crack lengths $a 0, a 1, a 2, \ldots, a 7$; the area near the current input and the upper right side of the compact tensile specimen (combination Q) is the optimal probe point. The distribution of probe points is shown in Figure 13. 
TABLE 2: Experimental equipment and model of crack monitoring.

\begin{tabular}{lccccc}
\hline \multirow{2}{*}{ Equipments } & Autoclave vessel & Stress-corrosion fatigue tensile machine & \multicolumn{3}{c}{ DCPD crack monitoring system } \\
& & & Nanovoltmeter & Power supply & Multiplex switch \\
\hline Models & YYF-30 & PLD-50 & Keysight 34420A & Keysight 6611C & Keysight 34970a \\
\hline
\end{tabular}

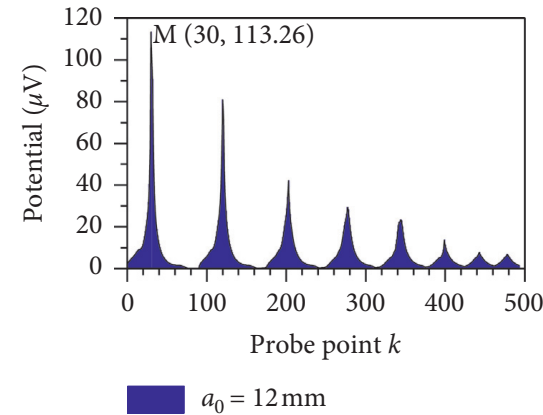

(a)

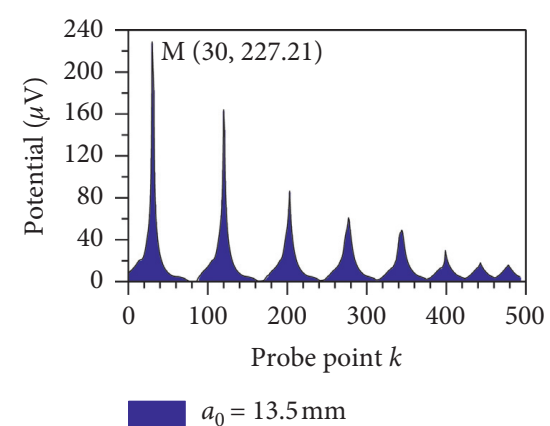

(d)

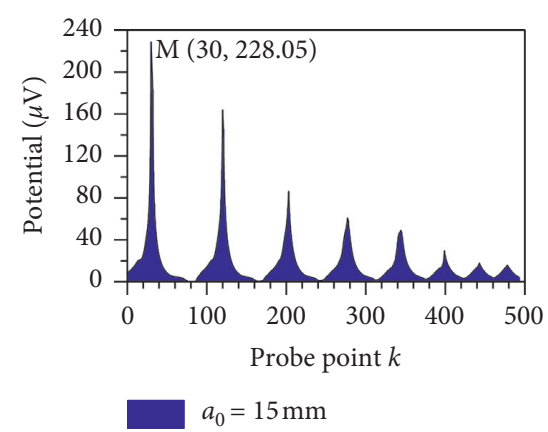

(g)

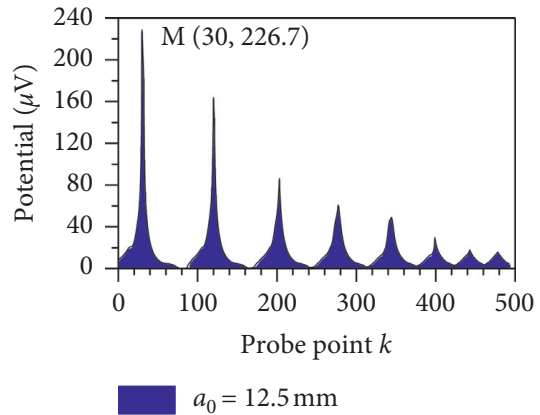

(b)

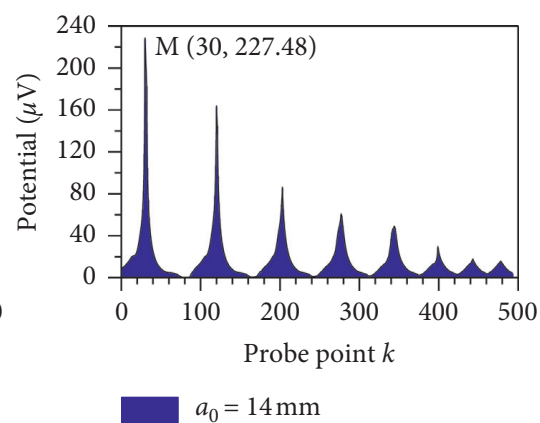

(e)

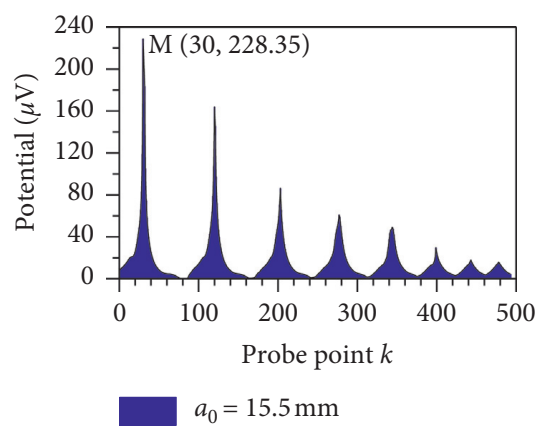

(h)

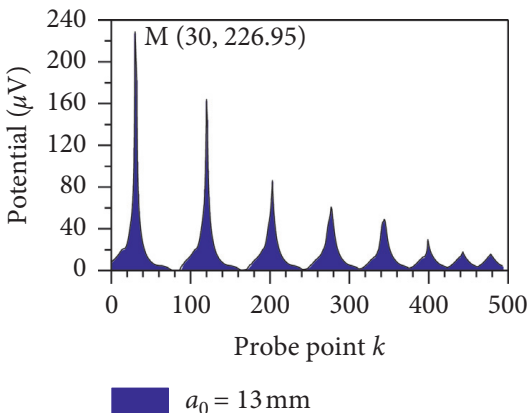

(c)

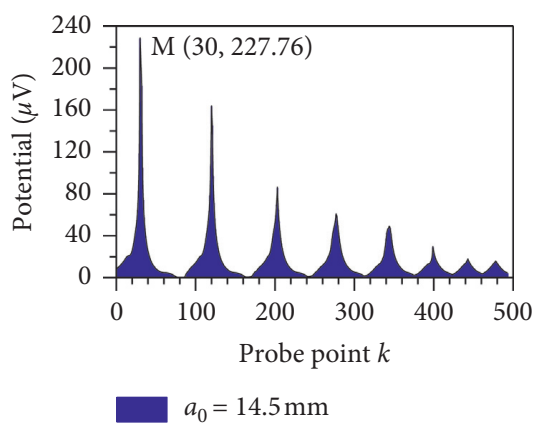

(f)

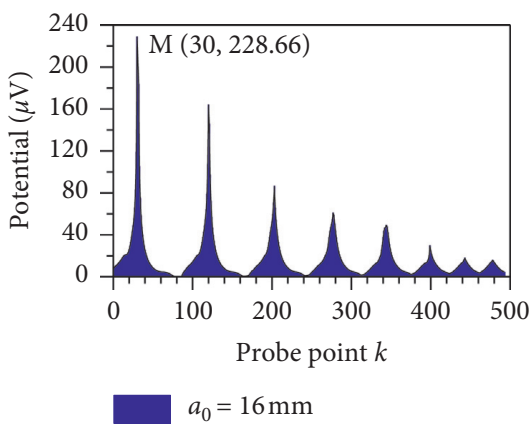

(i)

Figure 10: The distribution of the reference potential of the CT specimen.

5.3. Experimental Results. The experimental test results are shown as follows. Figure 14 presents, respectively, the numerical fluctuation of $\Delta U$ about crack independence. It can be obtained from the definition of crack independence that the smaller the $\Delta U$ value, the better the crack independence. By comparing the test data of crack independence, it can be obtained that the value of combination $\mathrm{N}$ in Figure 14(a) is the minimum and is near $9.7 E-6 \mu \mathrm{V}$, and the following is combination $\mathrm{Q}$; however, the value of combination $\mathrm{R}$ is the maximum. Since the values of combination $\mathrm{Q}$ and combination $\mathrm{N}$ are similar, therefore, the probe points with the optimal crack independence are combination $\mathrm{N}$ and combination $\mathrm{Q}$, and the probe points with the worst crack independence are combination $\mathrm{R}$.
According to the definition of measurability, the larger the value of $U_{n}$, the better the measurability. It can be seen from Figure 15 that when the probe points are located at combination $\mathrm{R}$, the value of $U_{n}$ regarding measurability is the maximum, and the following is combination Q; however, the value of combination $\mathrm{N}$ is the minimum, and consequently, the measurability of combination $\mathrm{R}$ and combination $\mathrm{Q}$ is much greater than the others, and the worst measurability is combination $\mathrm{N}$.

It can directly reflect the performance of the crack monitoring data from stability, accuracy, and resolution of the main potential. From Figure 16, it shows that when the probe points are combination $\mathrm{Q}$, the test value of the main 


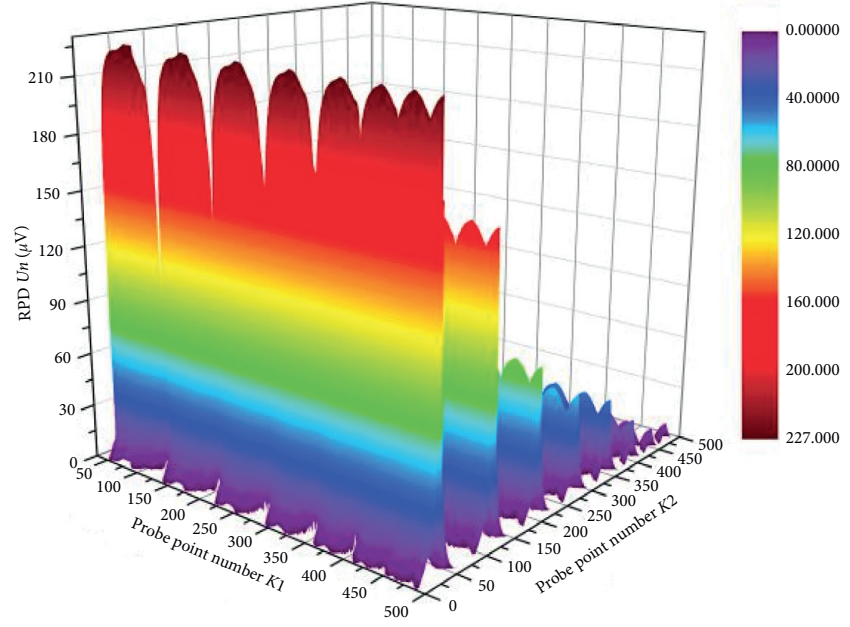

(a)

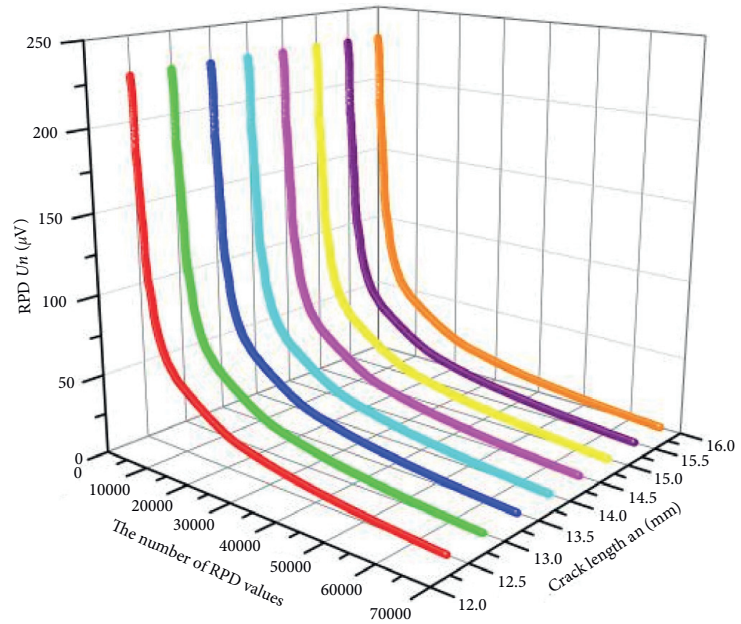

(b)

FIgURE 11: The optimization of measurability with GA. (a) The distribution of the RPD before GA optimization. (b) The distribution of the RPD after GA optimization.

TABLE 3: The value of the RPD after optimization of measurability.

\begin{tabular}{lccrc}
\hline Number $n$ & Crack length $a_{n}(\mathrm{~mm})$ & The value of RPD $U_{n}(\mu \mathrm{V})$ & Probe point $K 1(i, j)$ & Probe point $K 2(i, j)$ \\
\hline 65621 & 12.5 & 226.709 & $30(0,14)$ & $87(21,0) / 63(31,14)$ \\
65721 & 13 & 226.95 & $30(0,14)$ & $86(22,0) / 151(30,14)$ \\
65791 & 13.5 & 227.209 & $30(0,14)$ & $85(23,0) / 232(29,13)$ \\
65879 & 14 & 227.48 & $30(0,14)$ & $84(24,0) / 62(31,15)$ \\
65915 & 14.5 & 227.762 & $30(0,14)$ & $83(25,0) / 150(29,14)$ \\
65959 & 15 & 228.052 & $30(0,14)$ & $82(26,0) / 230(27,13)$ \\
65989 & 15.5 & 228.353 & $30(0,14)$ & $81(27,0) / 61(30,15)$ \\
66037 & 16 & 228.661 & $30(0,14)$ & $80(28,0) / 149(28,14)$ \\
\hline
\end{tabular}

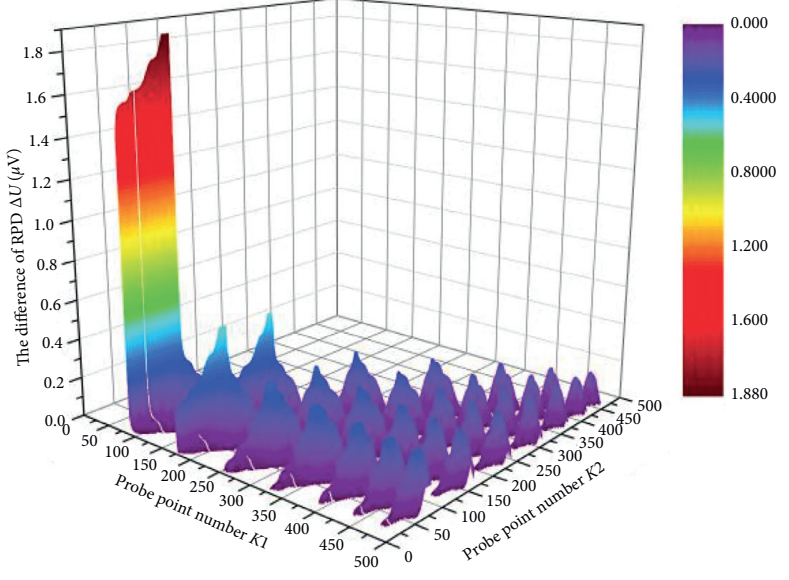

(a)

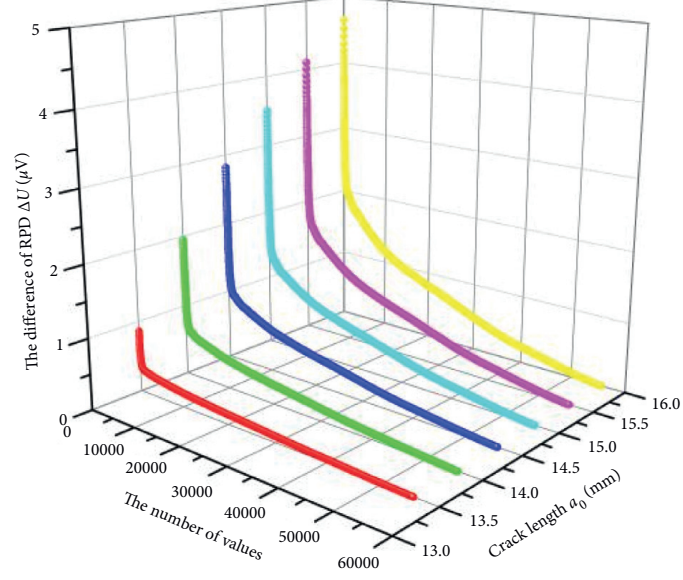

(b)

FIgURE 12: The optimization of crack independence with GA. (a) The difference between the RPD before optimization. (b) The difference between the RPD after optimization.

potential $U_{o}$ is larger and smoother than other values, and the fluctuation values of the adjacent data are less than $0.1 \mu \mathrm{V}$, which means that the reference potential has a strong compensation for the main potential and makes the monitoring signal of the instrument stable. Although combination $\mathrm{N}$ has the best crack independence and combination $\mathrm{R}$ has the best measurability, the potential of both is easily affected by crack growth; moreover, the 
TABLE 4: The difference between the RPD after optimization of crack independence.

\begin{tabular}{|c|c|c|c|c|c|}
\hline Number $n$ & $\begin{array}{l}\text { Crack length } a_{m} \\
(\mathrm{~mm})\end{array}$ & $\begin{array}{l}\text { Crack length } a_{n} \\
(\mathrm{~mm})\end{array}$ & $\begin{array}{l}\text { The difference between RPD } \Delta U \\
(\mu \mathrm{V})\end{array}$ & Probe point $K 1(i, j)$ & Probe point $K 2(i, j)$ \\
\hline 55330 & 12.5 & 13 & $1.21 E-05$ & $62(31,15)$ & $150(29,14)$ \\
\hline 55441 & 12.5 & 13.5 & $1.19 E-05$ & $228(25,13)$ & $367(24,12)$ \\
\hline 55226 & 12.5 & 14 & $1.16 E-05$ & $62(31,15)$ & $230(27,13)$ \\
\hline 55310 & 12.5 & 14.5 & $3.01 E-05$ & $62(31,15)$ & $230(27,13)$ \\
\hline 55044 & 12.5 & 15 & $3.14 E-05$ & $140(19,14)$ & $221(16,13)$ \\
\hline 55050 & 12.5 & 15.5 & $1.28 E-0.5$ & $66(0,13)$ & $370(27,11)$ \\
\hline 55075 & 12.5 & 16 & $1.09 E-05$ & $150(29,14)$ & $230(27,13)$ \\
\hline 55552 & 13 & 13.5 & $1.47 E-05$ & $52(0,14)$ & $141(6,15)$ \\
\hline 55320 & 13 & 14 & $1.26 E-05$ & $62(0,14)$ & $130(6,15)$ \\
\hline 55383 & 13 & 14.5 & $1.48 E-05$ & $140(19,14)$ & $221(16,13)$ \\
\hline 55200 & 13 & 15 & $1.24 E-05$ & $151(30,14)$ & $429(22,6)$ \\
\hline 55332 & 13 & 15.5 & $2.37 E-05$ & $215(12,13)$ & $408(15,8)$ \\
\hline 55128 & 13 & 16 & $2.69 E-05$ & $150(29,14)$ & $230(27,13)$ \\
\hline 55419 & 13.5 & 14 & $1.96 E-05$ & $62(31,15)$ & $230(27,13)$ \\
\hline 55527 & 13.5 & 14.5 & $2.58 E-05$ & $124(4,14)$ & $483(15,9)$ \\
\hline 55362 & 13.5 & 15 & $1.85 E-05$ & $291(15,12)$ & $430(21,5)$ \\
\hline 55475 & 13.5 & 15.5 & $2.59 E-05$ & $58(27,15)$ & $397(9,8)$ \\
\hline 55223 & 13.5 & 16 & $1.63 E-05$ & $150(29,14)$ & $230(27,13)$ \\
\hline 55699 & 14 & 14.5 & $1.32 E-05$ & $62(31,15)$ & $150(29,14)$ \\
\hline 55493 & 14 & 15 & $2.23 E-05$ & $150(29,14)$ & $230(27,13)$ \\
\hline 55581 & 14 & 15.5 & $2.93 E-05$ & $150(29,14)$ & $230(27,13)$ \\
\hline 55347 & 14 & 16 & $2.09 E-05$ & $291(15,12)$ & $451(17,10)$ \\
\hline 55587 & 14.5 & 15 & $1.65 E-05$ & $150(29,14)$ & $230(27,13)$ \\
\hline 55683 & 14.5 & 15.5 & $1.69 E-05$ & $150(29,14)$ & $230(27,13)$ \\
\hline 55524 & 14.5 & 16 & $2.07 E-05$ & $150(29,14)$ & $230(27,13)$ \\
\hline 55872 & 15 & 15.5 & $1.64 E-05$ & $153(30,12)$ & $370(27,11)$ \\
\hline 55702 & 15 & 16 & $3.01 E-05$ & $150(29,14)$ & $230(27,13)$ \\
\hline 55198 & 15.5 & 16 & $2.24 E-05$ & $52(0,14)$ & $150(29,14)$ \\
\hline
\end{tabular}

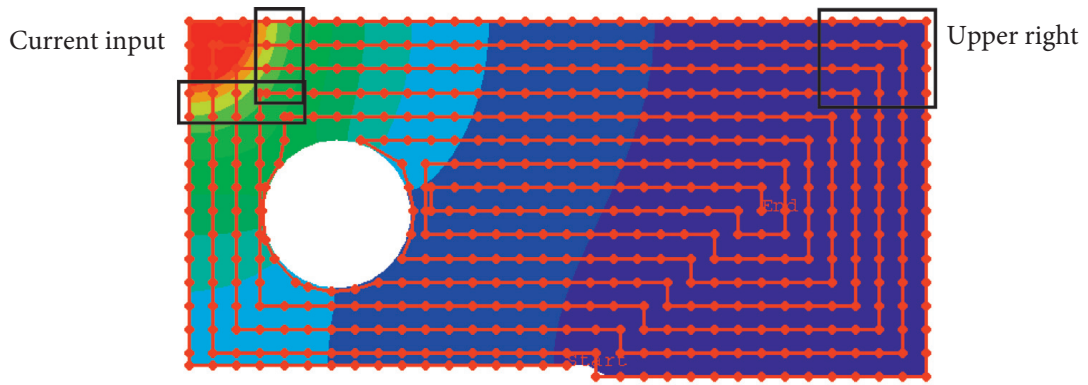

FIGURE 13: The optimal position of RPD probe points.

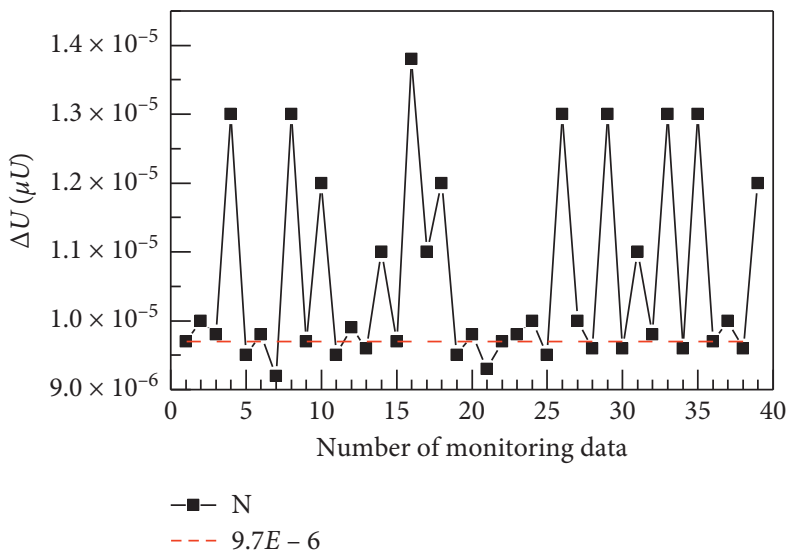

(a)

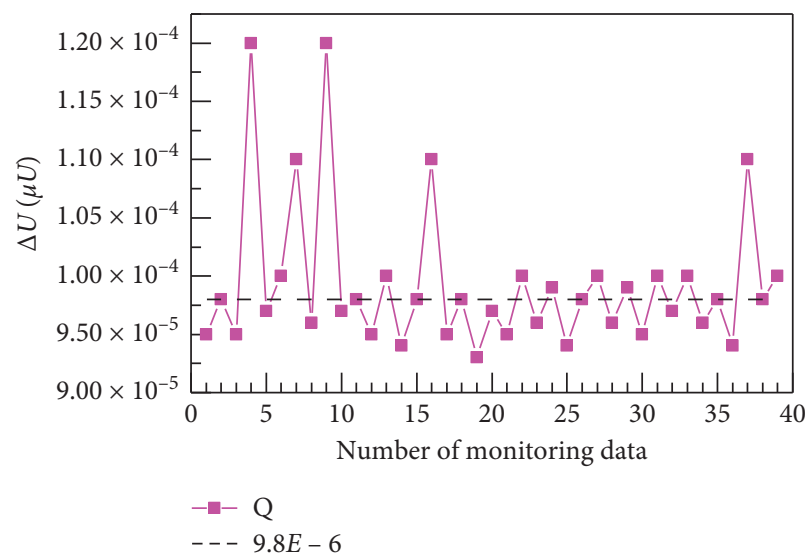

(b) 


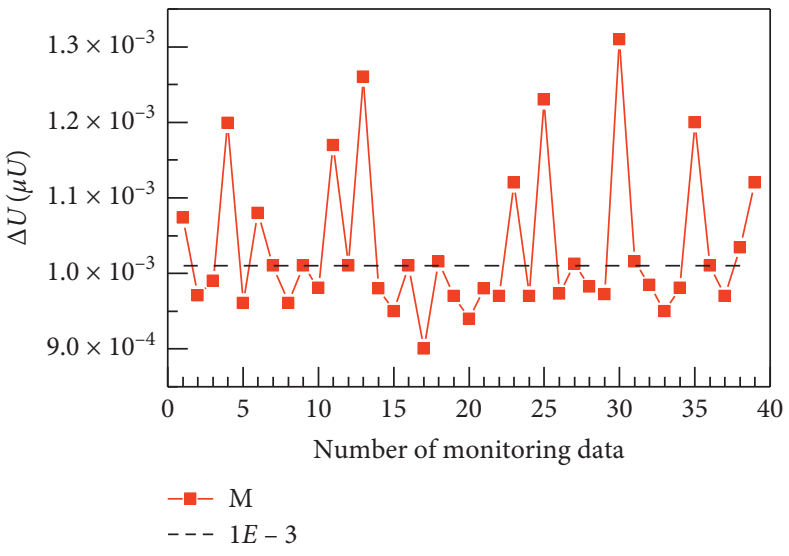

(c)

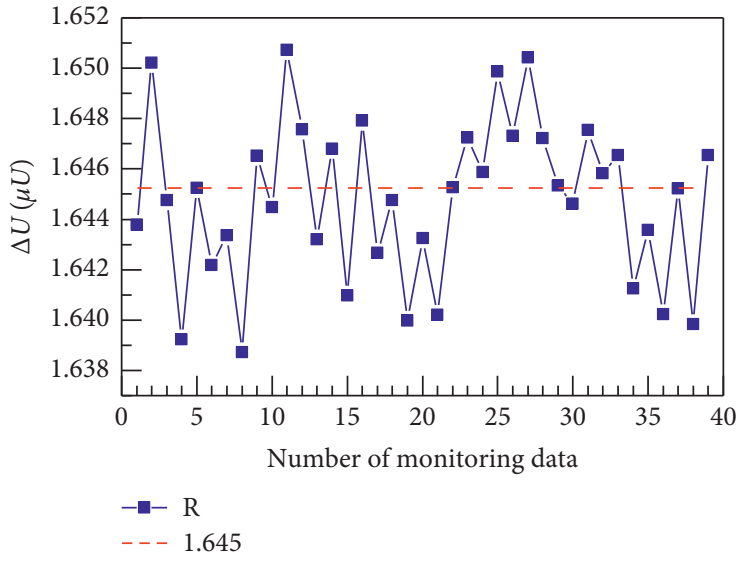

(d)

FIgURE 14: Test signal for crack independence.

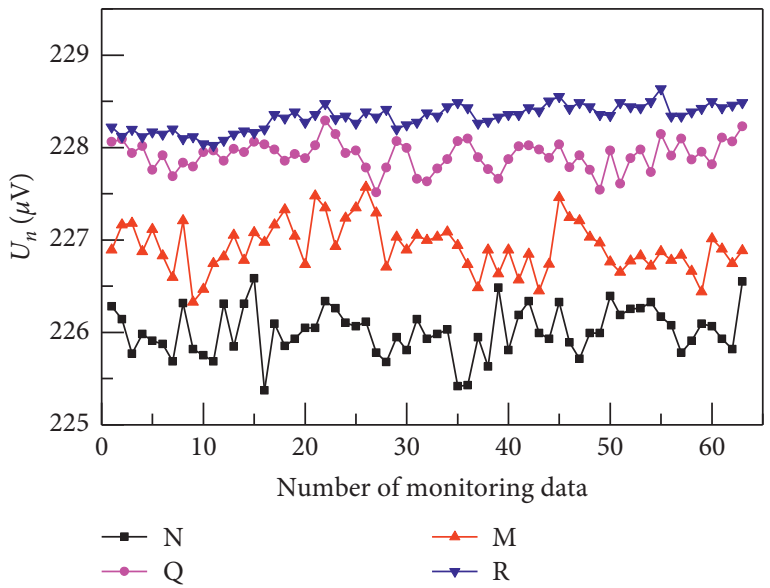

Figure 15: Test signal for measurability.

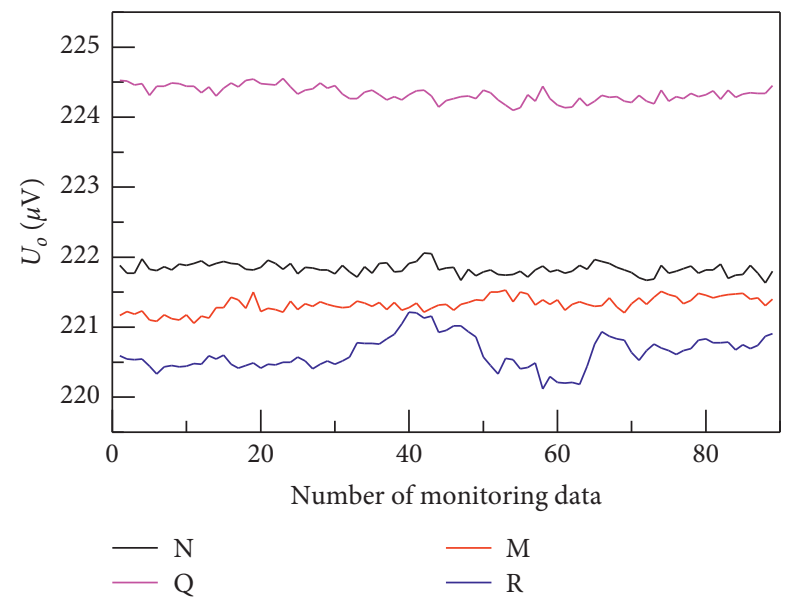

Figure 16: Test signal for the main potential.

reference potential difference cannot fully compensate the main potential difference. Eventually, considering comprehensively the stability and resolution of the main potential, as well as the measurability and crack independence of the reference potential, the position of the optimal probe points is near the current input and the upper right side of the specimen (combination $\mathrm{Q}$ ), which is the same as the optimized result of GA.

\section{Conclusions}

(1) In the process of crack monitoring by the DCPD method, the probe point position of the reference potential has a great impact on the crack monitoring signal. The finite element method combined with the genetic algorithm can obtain the accurate reference potential difference, which reduces the subjective factors and random errors and greatly compensates for the main potential difference; eventually, it ensures the reliability of the optimization results and improves the accuracy of the instrument monitoring.

(2) The measurability and the crack independence are related to the change of crack length. The longer the crack-growth length, the greater the reference potential difference and the better the measurability. The greater the difference between two different crack lengths, the greater the difference between the reference potential and the worse the crack independence.

(3) The experiment is performed under the condition that the material is 304 steel, the total length of the crack growth is $4 \mathrm{~mm}$, and the current input point is the upper left side of $0.5 \mathrm{~T}$-CT specimen. The optimal probe points are the area near the current input and the upper right side of the tensile specimen. This method provides a technical solution for optimizing the probe point position of the CT specimen at different current loading points and different crack lengths, as well as different materials. It also provides a theoretical basis for the reference potential method to deal 
with the temperature drift during the DCPD crack monitoring.

\section{Nomenclature}

$\begin{array}{ll}\text { SCC: } & \text { Stress corrosion cracking } \\ \text { CT: } & \text { Compact tensile samples } \\ \text { GA: } & \text { Genetic algorithm } \\ \text { RPD: } & \text { Reference potential difference } \\ \text { MPD: } & \text { Main potential difference } \\ \text { FEM: } & \text { Finite element method } \\ \text { DCPD: } & \text { Direct current potential drop } \\ \text { ACPD: } & \text { Alternating current potential drop } \\ \sigma_{0.2}: & \text { Yield strength, MPa } \\ E_{0}: & \text { Young's modulus, GPa } \\ v: & \text { Poisson's ratio } \\ \sigma: & \text { Conductivity, S/m. }\end{array}$

\section{Data Availability}

The data in the paper come from numerical simulations, algorithms, and experiments, without reference to others.

\section{Conflicts of Interest}

The authors declare no conflicts of interest.

\section{Acknowledgments}

This work was financially supported by the Natural Science Foundation of China (52075434) and Key Science and Technology Project of Shaanxi Provincial Science and Technology Department (2017GY-034).

\section{References}

[1] Z. Q. Lv, "Mechanisms and growth rate models for stress corrosion cracking in high temperature water," Materials China, vol. 7, pp. 651-662, 2019.

[2] V. Spitas, C. Spitas, and P. Michelis, "Real-time measurement of shear fatigue crack propagation at high-temperature using the potential drop technique," Measurement, vol. 41, no. 4, pp. 424-432, 2008.

[3] C. M. Omprakash, A. Kumar, M. Kamaraj, B. Srivathsa, and D. V. V. Satyanarayana, "Measurement of local creep strain in the notch region using AC potential drop technique," Measurement, vol. 145, pp. 500-502, 2019.

[4] M. Funk and J. Bär, "DCPD based detection of the transition from short to long crack propagation in fatigue experiments on the aluminum alloy 7475 T761," Procedia Structural Integrity, vol. 17, pp. 183-189, 2019.

[5] K. Zhao, H. Xue, L. Y. Zhao, and F. Yang, "Influence of randomness on mechanical properties at tip of stress corrosion cracking in nickel-based alloys," Rare Metal Materals and Engineering, vol. 48, pp. 3870-3875, 2019.

[6] I. Cerny, "The use of DCPD method for measurement of growth of cracks in large components at normal and elevated temperatures," Engineering Fracture Mechanics, vol. 71, no. 4-6, pp. 837-848, 2004.

[7] Z. M. Han, L. Hong, J. Meng et al., “Temperature drift modeling and compensation of capacitive accelerometer based on AGA-BP neural network," Measurement, vol. 164, p. 108019, 2020.

[8] S. Sinha, R. Bhardwaj, N. Sahu, H. Ahuja, R. Sharma, and R. Mukhiya, "Temperature and temporal drift compensation for $\mathrm{Al}_{2} \mathrm{O}_{3}$-gate ISFET-based $\mathrm{pH}$ sensor using machine learning techniques," Microelectronics Journal, vol. 97, p. 104710, 2020.

[9] M. A. Hicks and A. C. Pickard, "A comparison of theoretical and experimental methods of calibrating the electrical potential drop technique for crack length determination," International Journal of Fracture, vol. 20, no. 2, pp. 91-101, 1982.

[10] C. Q. Ni and H. Xue, "Analysis of the position of wiring points in DC potential drop crack monitoring test," Hot Processing Technology, vol. 45, pp. 122-125, 2016.

[11] M. Hu, "Measurement method of fatigue crack growth rate of three point bending specimen based on DCPD method," Journal of Shanghai Jiaotong University, vol. 12, pp. 17781784, 2015.

[12] A. Campagnolo, J. Bär, and G. Meneghetti, “Analysis of crack geometry and location in notched bars by means of a threeprobe potential drop technique," International Journal of Fatigue, vol. 124, pp. 167-187, 2019.

[13] A. Campagnolo, G. Meneghetti, F. Berto, and K. Tanaka, "Calibration of the potential drop method by means of electric $\mathrm{FE}$ analyses and experimental validation for a range of crack shapes," Fatigue \& Fracture of Engineering Materials \& Structures, vol. 41, no. 11, pp. 2272-2287, 2018.

[14] V. Spitas, C. Spitas, and P. Michelis, "A three-point electrical potential difference method for in situ monitoring of propagating mixed-mode cracks at high temperature," Measurement, vol. 43, no. 7, pp. 950-959, 2010.

[15] M. M. Devi and M. Geethanjali, "Hybrid of genetic algorithm and minimum spanning tree method for optimal PMU placements," Measurement, vol. 154, p. 107476, 2020.

[16] S. Wei, C. Zheng, and C. Lin, "Multi-objective optimization of cooling air distribution of grate cooler with different inlet temperatures by using genetic algorithm," Science China (Technological Sciences), vol. 60, pp. 345-354, 2017.

[17] G. Ekbatanifard and R. Monsefi, "A fast multi-objective genetic algorithm based approach for energy efficient QoSRouting in two-tiered wireless multimedia sensor networks," Modern Applied Science, vol. 4, no. 6, pp. 18-21, 2010.

[18] G. Ahn and S. Hur, "Efficient genetic algorithm for feature selection for early time series classification," Computers \& Industrial Engineering, vol. 142, p. 106345, 2020.

[19] Z. Q. Yang and B. Zhang, "Application of adaptive genetic algorithm in servo parameter optimization of linear motor feed system," Mechanical Science and Technology, vol. 1, no. 9, pp. 08-13, 2019, in Chinese.

[20] J. F. Gonçalves and G. Wäscher, "A MIP model and a biased random-key genetic algorithm based approach for a twodimensional cutting problem with defects," European Journal of Operational Research, vol. 286, no. 3, pp. 867-882, 2020.

[21] S. Liu and Z. Yi, "A method to enhance the activity of fewstate nodes based on genetic algorithm," Journal of Zhejiang University (Engineering Edition), vol. 53, no. 8, pp. 1546-1551, 2019, in Chinese.

[22] A. Gholami, H. Bonakdariau, I. Ebtehaj, M. Mohammadian, B. Gharabaghi, and S. R. Khodashenas, "Uncertainty analysis of intelligent model of hybrid genetic algorithm and particle swarm optimization with ANFIS to predict threshold bank profile shape based on digital laser approach sensing," Measurement, vol. 121, pp. 294-303, 2018. 